\title{
The peculiar (monic) polynomials, the zeros of which equal their
} coefficients

F. Calogero, F. Leyvraz

To cite this article: F. Calogero, F. Leyvraz (2017) The peculiar (monic) polynomials, the zeros of which equal their coefficients, Journal of Nonlinear Mathematical Physics 24:4, 545-555, DOI: https://doi.org/10.1080/14029251.2017.1375690

To link to this article: https://doi.org/10.1080/14029251.2017.1375690

Published online: 04 January 2021 


\title{
The peculiar (monic) polynomials, the zeros of which equal their coefficients
}

\author{
F. Calogero \\ Dipartimento di Fisica, Università di Roma "La Sapienza" and Istituto di Fisica Nucleare, Sezione di Roma, Rome, Italy \\ francesco.calogero@roma1.infn.it, francesco.calogero@uniroma1.it \\ F. Leyvraz* \\ Instituto de Física, Universidad Nacional Autónoma de México, Cuernavaca, Morelos 62210 México \\ f_leyvraz2001@hotmail.com
}

Received 21 May 2017

Accepted 12 June 2017

\begin{abstract}
We evaluate the number of complex monic polynomials, of arbitrary degree $N$, the zeros of which are equal to their coefficients. In the following, we call polynomials with this property peculiar polynomials. We further show that the problem of determining the peculiar polynomials of degree $N$ simplifies when any of the coefficients is either 0 or 1 . We proceed to estimate the numbers of peculiar polynomials of degree $N$ having one coefficient zero, or one coefficient equal to one, or neither.
\end{abstract}

Keywords: zeros of polynomials;Ulam polynomials

\section{Introduction}

Over half a century ago S.M. Ulam [1] discussed the transformation from $N$ numbers $x_{n}$ to their $N$ symmetrical sums $\sigma_{m}(\tilde{x})$ multiplied by $(-1)^{m}$,

$$
\begin{aligned}
y_{m} & =(-1)^{m} \sigma_{m}(\tilde{x}), \quad m=1, \ldots, N, \\
\sigma_{m}(\tilde{x}) & =\sum_{1 \leq n_{1}<n_{2}<\ldots<n_{m} \leq N} x_{n_{1}} x_{n_{2}} \cdots x_{n_{m}},
\end{aligned}
$$

implying, of course,

$$
\begin{aligned}
\sigma_{1}(\tilde{x}) & =x_{1}+\ldots+x_{N}, \\
\sigma_{N}(\tilde{x}) & =x_{1} \cdot \ldots \cdot x_{N} .
\end{aligned}
$$

Here $\tilde{x}$ is the unordered set of the $N$ numbers $x_{n}$ (see below).

It is well-known that the inversion of this transformation, (1.1), corresponds to the identification of the $N$ zeros of a polynomial of degree $N$ with coefficients $y_{m}$. While calling attention to this transformation "operating on the $N$-dimensional real space or on the $N$-dimensional complex space" [1] - Ulam pointed out the interest of investigating its fixed points, namely the identification of all the monic polynomials of degree $N$ with zeros $x_{n}$ equal to their coefficients $y_{m}$. Soon afterwards this problem was completely solved in the real domain-when the $N$ coefficients $y_{m}$ and the $N$ zeros $x_{n}$ are all real numbers-by Paul R. Stein [2]. He proved that in this real context—and

*Also at: Centro Internacional de Ciencias, Cuernavaca, Morelos 62210, México 
restricting attention only to polynomials with all zeros $x_{n}$, hence all coefficients $y_{m}$, nonvanishing (a well justified restriction, see below) - there are no polynomials of this kind with degree $N>4$. He moreover identified all the polynomials of this kind with degree $N \leq 4$ : for $N=2$ the single polynomial $(z-1)(z+2)=z^{2}+z-2$ (already mentioned by Ulam [1]); for $N=3$ the two polynomials $(z-1)(z+1)^{2}=z^{3}+z^{2}-z-1$ and $z^{3}+y_{1} z^{2}+y_{2} z+y_{3}$ with $y_{1}=w, y_{2}=1 / w$, $y_{3}=1 /(w+1)$ where $w \approx .5651977$ is the single real root of the cubic equation $2 w^{3}+2 w^{2}-1=0$; for $N=4$ the single polynomial $z^{4}+y_{1} z^{3}+y_{2} z^{2}+y_{3} z+y_{4}$ with $y_{1}=1, y_{2}=w \approx-1.7548777$, $y_{3}=1 / w \approx-0.5698403, y_{4}=y_{1} y_{2} y_{3}=1$, where $w$ is the single real root of the cubic equation $w^{3}+2 w^{2}+w+1=0$.

In this paper we treat the same problem but in the more general context of complex numbers. A motivation to do so is because this is generally a more natural context to discuss properties of polynomials since the complex numbers are algebraically closed. A second motivation is connected with the recently introduced notion of generations of (monic) polynomials, characterized by the property that the coefficients of the polynomials of degree $N$ of a generation coincide with (one of the $N$ ! permutations of) the zeros of the polynomials of the previous generation, see [3]. Earlier work on this subject includes, to the best of our knowledge, a paper by Di Scala and Maciá [4] proving that for any given degree, only finitely many such polynomials exist, as well as a paper by Bihun and Fulghesu [5] in which they independently derive some of the results we present here and further show that, among these polynomials, only the polynomial $z^{N}$ is an eigenfunction of a hypergeometric operator.

In the following Section 2 we review our notation and terminology and in Section 3 we report our main findings, which are then proven in Section 4. We present conclusions in Section 5.

\section{Notation and definitions}

\subsection{Notation}

Hereafter we suppose to work-unless otherwise indicated —with complex numbers and with monic polynomials of (positive integer) degree $N \geq 2$ in the complex variable $z$,

$$
p_{N}(z ; \vec{y}, \tilde{x})=z^{N}+\sum_{m=1}^{N} y_{m} z^{N-m}=\prod_{n=1}^{N}\left(z-x_{n}\right),
$$

which are characterized by their $N$ coefficients $y_{m}$ (the $N$ components of the $N$-vector $\vec{y}$ ) and by their $N$ zeros $x_{n}$ (the $N$ elements of the unordered set $\tilde{x}$ ). Of course above and hereafter indices such as $n, m$ are positive integers in the range from 1 to $N$ (unless otherwise indicated). The notation $p_{N}(z ; \vec{y}, \tilde{x})$, see $(2.2)$, is somewhat redundant, since this monic polynomial is equally well identified by assigning either its $N$ coefficients $y_{m}$ or its $N$ zeros $x_{n}$. Indeed its $N$ coefficients $y_{m}$ are explicitly expressed in terms of its $N$ zeros by the well-known formulas (1.1). Conversely, the unordered set $\tilde{x}$ of the $N$ zeros $x_{n}$ of the polynomial (2.2) is uniquely identified when the $N$ coefficients $y_{m}$ of this polynomial are assigned, although of course explicit formulas expressing the $N$ zeros $x_{n}$ in terms of the $N$ coefficients $y_{n}$ using radicals are generally only available for $N \leq 4$.

\subsection{Definitions}

The monic polynomial (2.2) is hereafter called peculiar if it has the peculiar property that its $N$ zeros $x_{n}$ can be ordered so as to coincide one-by-one with its $N$ coefficients $y_{m}$. We define $\mathscr{P}^{(N)}$ as 
the set of all such peculiar polynomials. In other words, $p_{N}(z) \in \mathscr{P}^{(N)}$ if and only if there exists a $\vec{y}$ such that

$$
p_{N}(z)=p_{N}(z ; \vec{y}, \vec{y}) .
$$

This set, as we shall see, can be divided into non-empty subsets: one is $\mathscr{P}_{0}{ }^{(N)}$, defined as the set of peculiar polynomials having at least one coefficient equal to 0 . Another is $\mathscr{P}_{1}{ }^{(N)}$, defined as the set of all peculiar polynomials having at least one coefficient equal to 1. Finally,let the set of the truly peculiar polynomials $\mathscr{P}_{t}^{(N)}$ be

$$
\mathscr{P}_{t}^{(N)}=\mathscr{P}^{(N)} \backslash\left(\mathscr{P}_{0}^{(N)} \cap \mathscr{P}_{1}^{(N)}\right),
$$

that is, the truly peculiar polynomials are those peculiar polynomials having no coefficient (nor, of course, zero) which vanishes or equals unity. The reason for this nomenclature is that, as we shall see below, whenever one of the coefficients of a peculiar polynomial is either 0 or 1 , the problem of identifying it can be reduced to one involving a lesser number of variables-i. e., polynomials of smaller degrees-whereas the elements of $\mathscr{P}_{t}^{(N)}$ are the truly new polynomials of degree $N$ having the property of being peculiar. As usual, for any set $\mathscr{S}$, we denote by $|\mathscr{S}|$ the number of elements of $\mathscr{S}$.

\section{Results}

In this section we state two results, a conjecture and a third result which holds conditionally on the conjecture. We provide the proofs in Section 4.

We begin by describing the structure of the set $\mathscr{P}^{(N)}$. First note the following obvious fact: any $p_{N}(z) \in \mathscr{P}_{0}^{(N)}$ can be written as

$$
p_{N}(z)=z p_{N-1}(z)
$$

where $p_{N-1}(z) \in \mathscr{P}^{(N-1)}$. There is therefore an elementary one-to-one correspondence between $\mathscr{P}_{0}^{(N)}$ and $\mathscr{P}^{(N-1)}$. There moreover holds

Proposition 3.1. For all $N \geq 3$, the set $\mathscr{P}^{(N)}$ can be divided in the following way

$$
\mathscr{P}^{(N)}=\mathscr{P}_{t}^{(N)} \cup\left(\mathscr{P}_{1}^{(N)} \backslash \mathscr{P}_{0}^{(N)}\right) \cup \mathscr{P}_{0}^{(N)},
$$

where the three sets $\mathscr{P}_{t}^{(N)}, \mathscr{P}_{1}^{(N)} \backslash \mathscr{P}_{0}^{(N)}$ and $\mathscr{P}_{0}^{(N)}$ are disjoint and non-empty.

Remark 3.1. Because of the correspondence between $\mathscr{P}_{0}^{(N)}$ and $\mathscr{P}^{(N-1)}$, see (3.1), we can proceed recursively to divide $\mathscr{P}_{0}^{(N)}$ further by applying Proposition 3.1 to $\mathscr{P}^{(N-1)}$.

Remark 3.2. Note that Proposition 3.1 fails when $N=2$, because, as we shall see below, $\mathscr{P}_{t}^{(2)}=\emptyset$.

Our second and main result concerns the number of peculiar polynomials of different types.

Proposition 3.2. For all $N \geq 3$ : 


$$
\begin{aligned}
\left|\mathscr{P}^{(N)}\right| & \leq N !, \\
\left|\mathscr{P}_{0}^{(N)}\right| & \leq(N-1) !, \\
\left|\mathscr{P}_{1}^{(N)}\right| & \leq(N-1) !, \\
\left|\mathscr{P}^{(N)} \backslash \mathscr{P}_{0}^{(N)}\right| & \leq(N-1)(N-1) !, \\
\left|\mathscr{P}_{1}^{(N)} \backslash \mathscr{P}_{0}^{(N)}\right| & \leq(N-2)(N-2) ! \\
\left|\mathscr{P}_{t}^{(N)}\right| & \leq\left(N^{2}-3 N+3\right)(N-2) !
\end{aligned}
$$

In the following instances the above inequalities are strict:

$$
\begin{aligned}
\left|\mathscr{P}_{1}^{(4)} \backslash \mathscr{P}_{0}^{(4)}\right|=3<4, & \\
\left|\mathscr{P}_{0}^{(N+1)}\right|= & \left|\mathscr{P}^{(N)}\right|<N ! \quad(N \geq 4), \\
& \left|\mathscr{P}_{1}^{(N)}\right|<(N-1) ! \quad(N \geq 4) .
\end{aligned}
$$

Remark 3.3. Note that the upper bounds (3.3b, 3.3e, 3.3f) on the number of elements of the 3 sets $\mathscr{P}_{0}^{(N)}, \mathscr{P}_{1}^{(N)} \backslash \mathscr{P}_{0}^{(N)}$ and $\mathscr{P}_{t}^{(N)}$ which build up $\mathscr{P}^{(N)}$ according to Proposition 3.1 add up to $N$ !:

$$
\left|\mathscr{P}_{0}^{(N)}\right|+\left|\mathscr{P}_{1}^{(N)} \backslash \mathscr{P}_{0}^{(N)}\right|+\left|\mathscr{P}_{t}^{(N)}\right| \leq N !
$$

consistently with (3.1) and (3.2). This implies that, if only one of these 3 sets has strictly fewer elements than what is given by the relevant upper bound given above, then the inequality (3.3a) for $\left|\mathscr{P}^{(N)}\right|$ will be strict, see (3.4b). Indeed, as follows from the special case (3.4a), this always happens whenever $N \geq 4$, implying (3.4b). The validity of the inequality (3.4c) is implied by an analogous argument. It is thus seen that the second and third strict inequalities $(3.4 \mathrm{~b}, 3.4 \mathrm{c})$ both follow from the first strict inequality (3.4a).

We now give the expressions for low-degree peculiar polynomials, which can be obtained by solving the equations presented in Section 4 via such a program as Mathematica [6]. Since the polynomials of $\mathscr{P}_{0}^{(N)}$ can easily be obtained from the polynomials belonging to the sets $\mathscr{P}_{1}^{(M)} \backslash \mathscr{P}_{0}^{(M)}$ and $\mathscr{P}_{t}^{(M)}$ with $M<N$, we only give the polynomials belonging to the sets $\mathscr{P}_{1}^{(N)} \backslash \mathscr{P}_{0}^{(N)}$ and $\mathscr{P}_{t}^{(N)}$. For $N \leq 4$ these peculiar polynomials are as follows:

For $N=2: \mathscr{P}_{t}^{(2)}=\emptyset$ and there is 1 polynomial $z^{2}+z-2=(z-1)(z+2)$ belonging to $\mathscr{P}_{1}^{(2)} \backslash \mathscr{P}_{0}^{(2)}$.

For $N=3$ : there is 1 polynomial $z^{3}+z^{2}-z-1=(z-1)(z+1)^{2} \in \mathscr{P}_{1}^{(3)} \backslash \mathscr{P}_{0}^{(3)}$ and 3 polynomials $z^{3}+y_{1} z^{2}+y_{2} z+y_{3} \in \mathscr{P}_{t}^{(3)}$ where

$$
\begin{aligned}
& y_{1}=w, \\
& y_{2}=-1-2 w+2 w^{3}, \\
& y_{3}=-1-2 w^{3},
\end{aligned}
$$


the number $w$ being one of the 3 roots of the cubic equation $2 w^{3}+2 w^{2}-1=0$ (only one of which is real, see above). Note that this equation is irreducible over the rationals, a fact we shall make use of later. In particular, note that here and throughout the following, whenever we speak of an irreducible polynomial, we shall always implicitly mean this to be understood over the rationals.

For $N=4$ : there are altogether 17 polynomials in $\mathscr{P}^{(4)} \backslash \mathscr{P}_{0}^{(4)}$, of which 3 read as follows: $z^{4}+z^{3}+y_{2} z^{2}+y_{3} z+y_{4} \in \mathscr{P}_{1}^{(4)} \backslash \mathscr{P}_{0}^{(4)}$, where

$$
\begin{aligned}
& y_{2}=w \\
& y_{3}=-w^{3}-3 w^{2}-3 w-2, \\
& y_{4}=w\left(w^{2}+3 w+2\right),
\end{aligned}
$$

and $w$ is one of the 3 roots of the irreducible cubic equation

$$
w^{3}+2 w^{2}+w+1=0 .
$$

Again, note that only 1 root of this cubic is real (see above).

The remaining 14 polynomials, none of which is real, belong to $\mathscr{P}_{t}^{(4)}$ and are of the form

$$
z^{4}+y_{1} z^{3}+y_{2} z^{2}+y_{3} z+y_{4}
$$

where

$$
\begin{aligned}
& y_{1}=w \\
& y_{2}=\frac{1}{3301}\left(-5780-9301 w-15701 w^{2}-19444 w^{3}\right. \\
& \quad+15074 w^{4}+62196 w^{5}+79384 w^{6}+62708 w^{7} \\
& \quad+7240 w^{8}-87856 w^{9}-157888 w^{10}-149344 w^{11} \\
&\left.\quad-79664 w^{12}-17888 w^{13}\right), \\
& y_{3}=\frac{1}{3301}\left(2950-7255 w-24398 w^{2}-24629 w^{3}\right. \\
& \quad+30824 w^{4}+51850 w^{5}+60560 w^{6}+34348 w^{7} \\
& \quad-35540 w^{8}-129696 w^{9}-116400 w^{10}-27936 w^{11} \\
& \quad+78384 w^{12}+69808 w^{13}+30528 w^{14}-9903 y_{2} \\
&\left.\quad-9903 y_{2}^{2}-3301 y_{2}^{3}\right), \\
& 1 \\
& y_{4}=\frac{1}{3301}\left(-2950+653 w+24398 w^{2}+24629 w^{3}\right. \\
& \quad-30824 w^{4}-51850 w^{5}-60560 w^{6}-34348 w^{7} \\
& \quad+35540 w^{8}+129696 w^{9}+116400 w^{10}+27936 w^{11} \\
& \quad-78384 w^{12}-69808 w^{13}-30528 w^{14}+6602 y_{2} \\
&\left.\quad+9903 y_{2}^{2}+3301 y_{2}^{3}\right),
\end{aligned}
$$

where $w$ is one of the 14 roots-all distinct and complex, consisting of 7 complex conjugate pairsof the following irreducible polynomial equation of degree 14 :

$$
\begin{aligned}
& 1+3 w+6 w^{2}+6 w^{3}+3 w^{4}-12 w^{5}-34 w^{6}-44 w^{7} \\
& \quad-28 w^{8}+4 w^{9}+48 w^{10}+80 w^{11}+80 w^{12}+48 w^{13}+16 w^{14}=0 .
\end{aligned}
$$


This completes our treatment of the $N=4$ case.

Note the remarkable way these findings, valid in the complex context, extend those found by Stein in the real case (as reported in Section 2 above). For $N>4$ the extension to the complex case of Stein's findings for the real case is even more significant, see below, but the results become too unwieldy to permit their explicit display.

We have seen so far that $\mathscr{P}^{(N)}$ can be divided into simpler subsets, each of which can be brought into one-to-one correspondence, using (3.1), with sets of the type $\mathscr{P}_{t}^{(M)}$ and $\mathscr{P}_{1}^{(M)} \backslash \mathscr{P}_{0}^{(M)}$ for $M \leq N$. We may ask whether the subdivision can go further. That this will not happen for all $N$ is immediately clear from the results presented above for $2 \leq N \leq 4$.

Indeed, for all these values of $N$, we find that the sets $\mathscr{P}_{t}^{(N)}$ and $\mathscr{P}_{1}^{(N)} \backslash \mathscr{P}_{0}^{(N)}$ are irreducible over $\mathbb{Q}$ in the following sense: $y_{1}$ can, in all cases, be expressed as the root of an appropriate polynomial $\Pi_{1}(y)$ with rational coefficients, irreducible over $\mathbb{Q}$. The $y_{k}$ for $2 \leq k \leq N$ are then given as polynomials of $y_{1}$. Clearly, the peculiar role of $y_{1}$ is only apparent: for any $k$, with $1 \leq k \leq N$, the $y_{l}$ with $l \neq k$ can then also be expressed in terms of $y_{k}$, which in turn is then the root of an appropriate irreducible polynomial $\Pi_{k}(y)$. Clearly, if the polynomial $\Pi_{1}(y)$ which defines a given set of polynomials, were reducible, we could factorize it into polynomials with integer coefficients. This would then allow to divide this subset further according to whether $y_{1}$ is a zero of one or the other of the factors. Since the $\Pi_{1}(y)$ in all the cases described above, are in fact irreducible, such an additional reduction of the subsets described above is not possible.

In the following, we shall show that all elements of $\mathscr{P}_{1}^{(N)} \backslash \mathscr{P}_{0}^{(N)}$ satisfy the system of equations

$$
\begin{aligned}
y_{2}+\ldots+y_{N} & =-2, \\
\sigma_{m}\left(1, y_{2}, \ldots, y_{N}\right) & =(-1)^{m} y_{m}, \quad(2 \leq m \leq N-2), \\
y_{2} \cdot \ldots \cdot y_{N-1} & =(-1)^{N} .
\end{aligned}
$$

Similarly, we shall see that the elements of $\mathscr{P}_{t}^{(N)}$ all satisfy the system of equations:

$$
\begin{aligned}
& \sigma_{m}(\vec{y})=(-1)^{m} y_{m} \quad(1 \leq m \leq N-2), \\
& y_{1} \cdot \ldots \cdot y_{N-1}=(-1)^{N}, \\
& 2 y_{1}\left(\sum_{l=0}^{N-2} y_{1}^{l}\right)+\sum_{k=1}^{N-1} y_{k}\left(\sum_{l=0}^{N-(k+1)} y_{1}^{l}\right)=0 .
\end{aligned}
$$

From standard elimination theory, as described, for example, in [7], one may show that the solutions of equations such as (3.10) and (3.11), can be described by saying that $y_{1}$ is the solution of a polynomial equation, whereas the $y_{k}$ for $2 \leq k \leq N$ are expressed via a polynomial relation connecting $y_{k}$ and $y_{1}$. The differences with the characterizations above are twofold: the polynomial need not be irreducible and the connection between $y_{k}$ and $y_{1}$ is a general polynomial equation, whereas above we had noticed that $y_{k}$ can be expressed as a polynomial expression of $y_{1}$.

We now formulate the

Conjecture 3.1. For all $N \geq 5$, upon reducing (via standard elimination theory, as above) the systems of equations (3.10) and (3.11), $y_{1}$ can be expressed as the zero of a polynomial $\Pi_{1}(y)$, irreducible, and the $y_{k}$ 's can be expressed as polynomials of $y_{1}$. 
Remark 3.4. Note that, for $N=4$, the total set of polynomials belonging to $\mathscr{P}_{1}^{(4)} \backslash \mathscr{P}_{0}^{(4)}$ can be characterized by an irreducible polynomial as stated above, see (3.10). On the other hand, the Conjecture does not hold in this case, because it is found that then a solution of (3.10) with $y_{4}=0$ exists. Indeed, this is the exception which causes all the special cases identified above (in the last part of Proposition 3.2).

There then holds the following result:

Proposition 3.3. If the Conjecture holds, then inequalities (3.3a, 3.3b, 3.3c) of Proposition 3.2 hold as equalities for $N \geq 5$, so that the cases of strict inequality (3.4) —as stated in the last part of Proposition 3.2-are the only possible ones.

We consider the Conjecture plausible but we recognize that the arguments for its validity are so far limited to the cases we tested numerically via Mathematica [6]: $N \leq 7$ for (3.10) and $N \leq 6$ for (3.11).

\section{Proofs}

The coefficients $y_{n}$ of a peculiar polynomial $p_{N}(z) \in \mathscr{P}^{(N)}$ clearly satisfy the set of equations

$$
\sigma_{m}\left(y_{1}, \ldots, y_{N}\right)=(-1)^{m} y_{m}, \quad(1 \leq m \leq N) .
$$

Here and below $\sigma_{m}\left(y_{1}, \ldots, y_{N}\right)$ is of course defined by (1.1b) with the $N$ zeros $x_{n}$ replaced by the $N$ coefficients $y_{m}$. These equations can be reformulated so as to be equations in projective space, as follows

$$
\sigma_{m}\left(y_{1}, \ldots, y_{N}\right)=(-1)^{m} y_{0}^{m-1} y_{m}, \quad(1 \leq m \leq N) .
$$

To find the full number of solutions of (4.2) when counted with the appropriate multiplicities, we apply Bézout's theorem (see, for instance, [8] for a statement and a proof; those not familiar with commutative algebra may find the arguments in [7] easier to follow). Since, as it is readily verified, the only solution with $y_{0}=0$ is the trivial solution where all $y_{n}$ vanish, the solutions of (4.2) are all solutions of (4.1). The number of solutions of (4.1) counted in the same manner is therefore the product of the degrees of all these equations (4.1), that is, $N$ !. Since all elements of $\mathscr{P}^{(N)}$ satisfy (4.1) and since multiplicities are always larger or equal to one, the validity of inequality (3.3a) of Proposition 3.2 is thereby demonstrated.

Note in passing that the coefficients $y_{n}$ of a peculiar polynomial $p_{N}(z)$ must, of course, satisfy the $N$ algebraic equations

$$
p_{N}\left(y_{m}\right)=y_{m}^{N}+\sum_{r=1}^{N} y_{r} y_{m}^{N-r}=0, \quad(1 \leq m \leq N) .
$$

However, contrary to (4.2), these equations do not guarantee that the corresponding polynomial be peculiar, because they do not imply that all zeros of the polynomial $p_{N}(z)$ are coefficients. A counterexample is for instance given by the polynomial $p_{2}(z)=z^{2}-z / 2-1 / 2$, the coefficients of which satisfy (4.3), but which is not peculiar, because $z=1$ is a zero of $p_{2}(z)$ without being one of 
its coefficients. For future use, we point out the projective form of (4.3)

$$
y_{m}^{N}+\sum_{r=1}^{N} y_{0}^{r-1} y_{r} y_{m}^{N-r}=0, \quad(1 \leq m \leq N) .
$$

Let us now consider the set $\mathscr{P}_{0}^{(N)}$, i. e. the case in which one of the coefficients vanishes, say $y_{k}=0$. It then follows from (4.2) with $m=N$ that $y_{N}$ vanishes, $y_{N}=0$. From this follows that (4.2) for $m=N$ is an identity, and can thus be discarded. The system (4.2) then becomes a system of $N-1$ equations in the $N-1$ unknowns, $y_{1}, \ldots, y_{N-1}$, to which we may again apply Bézout's theorem. It thus follows that, again counting multiplicities, there are $(N-1)$ ! solutions to this system of equations. Since each peculiar polynomial $p_{N}(z) \in \mathscr{P}_{0}^{(N)}$ satisfies this system of equations, and since multiplicities are always larger than or equal to one, this proves inequality (3.3b) of Proposition 3.2. Note that this result could also have been obtained from the simple remark

$$
\left|\mathscr{P}_{0}^{(N)}\right|=\left|\mathscr{P}^{(N-1)}\right|
$$

and inequality (3.3a) applied with $N$ replaced by $N-1$.

Let us next consider the set $\mathscr{P}_{1}^{(N)}$, i. e. the case in which one of the coefficients equals unity, say $y_{k}=1$, which in the projective formulation amounts to saying $y_{k}=y_{0}$. We first show that then $y_{1}=y_{0}$. To this end, we consider (4.4) for the case in which $m=k$. It then follows from $y_{k}=y_{0}$ that

$$
y_{0}+\sum_{r=1}^{N} y_{r}=0
$$

Comparing this with (4.2) for $m=1$ we immediately obtain that $y_{1}=y_{0}$.

We may now replace $y_{1}$ by $y_{0}$ in all the equations (4.2). This yields

$$
\begin{aligned}
\sigma_{1}\left(y_{0}, y_{2}, \ldots, y_{N}\right) & =-y_{0} \\
\sigma_{m}\left(y_{0}, y_{2}, \ldots, y_{N}\right) & =(-1)^{m} y_{0}^{m-1} y_{m} \quad(2 \leq m \leq N) .
\end{aligned}
$$

This system of $N$ algebraic equations can clearly be rewritten as follows:

$$
\begin{aligned}
& \sigma_{1}\left(0, y_{2}, \ldots, y_{N}\right)=-2 y_{0}, \\
& (-1)^{m}\left[y_{0} \sigma_{m-1}\left(0, y_{2}, \ldots, y_{N}\right)+\sigma_{m}\left(0, y_{2}, \ldots, y_{N}\right)\right]=y_{0}^{m-1} y_{m}, \\
& (-1)^{N} \sigma_{N-1}\left(0, y_{2}, \ldots, y_{N}\right)=y_{0}^{N-2} y_{N},
\end{aligned}
$$

where, in (4.8b), $2 \leq m \leq N-2$. We now combine equations (4.8b) with each other for all values of $m$ and, via (4.8a), we obtain the last, (4.8c); which is thus seen to be superfluous. The equations satisfied by the coefficients of a polynomial belonging to $\mathscr{P}_{1}^{(N)}$ thus reduce to a set of $N-1$ equations for the $N-1$ unknowns $y_{2}, \ldots, y_{N}$. We therefore have described $\mathscr{P}_{1}^{(N)}$ by $N-1$ equations, the degrees of which have a product of $(N-1)$ !. The coefficients of a polynomial $p_{N}(z) \in \mathscr{P}_{1}^{(N)}$ are thus characterized by the fact that they satisfy a system of equations which has $(N-1)$ ! solutions counted with multiplicity. It thus follows, as stated by inequality (3.3c) of Proposition 3.2, that the total number of such polynomials cannot exceed $(N-1)$ !.

Note that we have shown that the sets $\mathscr{P}^{(N)}, \mathscr{P}_{0}^{(N)}$, and $\mathscr{P}_{1}^{(N)}$ correspond exactly to the solutions of some appropriate system of algebraic equations. That is, a polynomial belongs to one of these 
sets if and only if its coefficients satisfy the corresponding set of equations. The difference between the number of elements of these sets and their upper bounds reported in Proposition 3.2 can thus only arise from the existence of multiple solutions in the equations derived above for each one of these 3 sets. This will not hold any more for the sets we consider in the following.

Since we have, up to now, only obtained upper bounds for the cardinalities of $\mathscr{P}^{(N)}, \mathscr{P}_{0}^{(N)}$ and $\mathscr{P}^{(N)}$, there does not follow any non-trivial estimate either from below or from above, say, for $\left|\mathscr{P}^{(N)} \backslash \mathscr{P}_{0}^{(N)}\right|$. To obtain these, we proceed to determine equations which the elements of such sets must satisfy. These will always provide an upper bound on the number of elements in the set. However, we will not generally be able to show that all the solutions of the equations belong to the set, but only the converse: all elements of the set do satisfy the equations.

The simplest example is given by $\mathscr{P}^{(N)} \backslash \mathscr{P}_{0}^{(N)}$ : in this case we may simplify the equation (4.2) to

$$
\begin{aligned}
\sigma_{m}\left(y_{1}, \ldots, y_{N}\right) & =(-1)^{m} y_{0}^{m-1} y_{m}, \quad(1 \leq m \leq N-1) \\
y_{1} \cdot \ldots \cdot y_{N-1} & =(-1)^{N} y_{0}^{N-1} .
\end{aligned}
$$

(4.9b) was obtained by dividing both sides of the equation (4.2) corresponding to $m=N$ by $y_{N}$, which requires, but does not imply, $y_{N} \neq 0$. The degree of this equation has thus decreased by one. Via Bézout's theorem we may then deduce that the corresponding system of equations has exactly $(N-1)(N-1)$ ! solutions counted with multiplicity, and hence, as above, that $\left|\mathscr{P}^{(N)} \backslash \mathscr{P}_{0}^{(N)}\right| \leq$ $(N-1)(N-1)$ !, as stated by inequality (3.3d) of Proposition 3.2.

Note that one of the special cases in which the inequalities are strict, see (3.4a), arises precisely from such an instance: when $N=4$, the set of equations (4.9) has among its solutions the following values of $y_{n}: y_{1}=1, y_{2}=-1, y_{3}=-1$ and $y_{4}=0$. There is thus an element of $\mathscr{P}_{0}^{(4)}$ among the solutions of the above equations. Note that this immediately implies that the corresponding zero is a double zero of the set of equations (4.2), so that inequality (3.3a) of Proposition 3.2 is then also strict.

Similarly, we may consider the case of $\mathscr{P}_{1}^{(N)} \backslash \mathscr{P}_{0}^{(N)}$. The set $\mathscr{P}_{1}^{(N)}$, as we have seen, is described by (4.8) of which the final one is superfluous. However, we can equally well discard the second equation (4.8b) corresponding to $m=N-1$. If we then use the assumption $y_{N}=0$ in order to divide by $y_{N}$ in the final equation (4.8c), we are led to a system of $N-2$ equations, the orders of which have a product of $(N-2)$ !, complemented by one final equation of order $N-2$. These correspond exactly to (3.10). Since all elements of $\mathscr{P}_{1}^{(N)} \backslash \mathscr{P}_{0}^{(N)}$ satisfy these equations, the result claimed in inequality (3.3e) of Proposition 3.2 is shown.

To estimate $\left|\mathscr{P}_{t}^{(N)}\right|$, we proceed as follows: first, we note that the elements of $\mathscr{P}^{(N)} \backslash \mathscr{P}_{0}^{(N)}$ satisfy the equations

$$
\begin{aligned}
\sigma_{m}\left(y_{1}, \ldots, y_{N}\right) & =(-1)^{m} y_{0}^{m-1} y_{m}, \quad(1 \leq m \leq N-1), \\
y_{1} \cdot \ldots \cdot y_{N-1} & =(-1)^{N} y_{0}^{N-1} .
\end{aligned}
$$

Let us now ask, within this set, how many elements satisfy $y_{1}=y_{0}$. As above, we show that these solutions satisfy the equations: 


$$
\begin{aligned}
\sigma_{1}\left(y_{0}, y_{2}, \ldots, y_{N}\right) & =-y_{0}, \\
\sigma_{m}\left(y_{0}, y_{2}, \ldots, y_{N}\right) & =(-1)^{m} y_{0}^{m-1} y_{m}, \quad(2 \leq m \leq N-2), \\
y_{2} \cdot \ldots \cdot y_{N-1} & =(-1)^{N} y_{0}^{N-2} .
\end{aligned}
$$

Note that, here as in (4.8), we show that the equation for $m=N-1$ follows from the others and can thus be discarded. From Bézout's theorem follows that (4.10) have $(N-1)^{2}(N-2)$ ! solutions, whereas $(4.11)$ have $(N-2)(N-2)$ ! solutions, both counted according to multiplicities. Now the solutions of (4.11) are a subset of those of (4.10). They thus have at most the same multiplicities. It thus follows that $\left|\mathscr{P}_{t}^{(N)}\right| \leq\left[(N-1)^{2}-(N-2)\right](N-2)$ !, or in other words $\mathscr{P}_{t}^{(N)} \leq\left(N^{2}-3 N+\right.$ $3)(N-2)$ ! as stated in inequality (3.3f) of Proposition 3.2.

We can also deduce that all elements of $\mathscr{P}_{t}^{(N)}$ satisfy (3.11): to this end we perform the following operations on (4.1): divide the equation of (4.1) corresponding to $m=N$ by $y_{N}$, using the fact that $y_{N} \neq 0$. Then replace the equation corresponding to $m=N-1$ by the equation (4.4) for $m=1$. Finally, subtract equation (4.1) for $m=1$ from that last equation, which may then be divided by $y_{1}-$ 1. The resulting equations are then exactly (3.11). Note that the projective form of these equations, that is, the equations (3.11) modified so as to be homogeneous in the $y_{m}$ 's via the introduction of the parameter $y_{0}$, have a number of solutions "at infinity", that is, corresponding to $y_{0}=0$. Since Bézout's theorem yields the number of solutions for the projective form of the equations, it follows that the number of solutions of (3.11) is strictly less than the number obtained via Bézout's theorem, namely $(N-1)(N-1)$ !, as indeed corresponds to the statement of (3.3f).

Finally, we need to prove Proposition 3.3. Assuming the Conjecture, we see that neither (3.10) nor (3.11) can have multiple solutions, since an irreducible polynomial does not have multiple zeros. It remains to see, then, that neither (3.10) nor (3.11) can have solutions which do not correspond to elements of $\mathscr{P}_{1}^{(N)} \backslash \mathscr{P}_{0}^{(N)}$, or elements of $\mathscr{P}_{t}^{(N)}$ respectively. Let us begin with the first case, the second being quite similar.

Let $\left(y_{2}, \ldots, y_{N}\right)$ be a solution of (3.10). Then it is clear that the polynomial $z^{N}+z^{N-1}+$ $\sum_{k=2}^{N} y_{k} z^{N-k}$ is peculiar, and has a coefficient $y_{1}=1$. It thus merely remains to show that $y_{N} \neq 0$. From (3.10) and $y_{N}=0$ follows, combining the equation for $m=N-2$ and the last equation, that $y_{N-1}=-1$. According to the conjecture, however, the solution set of (3.10) can be described by stating that $y_{N-1}$ is the root of an appropriate polynomial, irreducible over $\mathbb{Q}$, and that the $y_{k}$ with $k \neq N-1$ are expressed by polynomials in $y_{N-1}$. Now the very fact that $y_{N-1}=-1$ contradicts irreducibility. One shows quite similarly that (3.11) can have no solutions with $y_{N}=0$, again because in that case $y_{N-1}=-1$. It therefore follows that $\left|\mathscr{P}_{1}^{(N)} \backslash \mathscr{P}_{0}^{(N)}\right|$ and $\left|\mathscr{P}_{t}^{(N)}\right|$ are equal to $(N-2)(N-2)$ ! and $\left(N^{2}-3 N+3\right)(N-2)$ !, respectively.

\section{Conclusions}

Summarizing, we have shown that peculiar polynomials, defined as those polynomials which remain identical when we replace their coefficients by (an appropriate permutation of) their zeros, exist for all degrees $N$. The set of such polynomials can be divided into three disjoint sets, namely those which have one coefficient equal to 0 , those which have no coefficient equal to 0 , but at least one equal to 1 , and finally those which have no coefficients equal to either 1 or 0 . The first of these three sets can further be divided, since each of its elements corresponds to a peculiar polynomial 
of degree $N-1$. We further show that, if we consider complex solutions, the above sets are all non-empty for all $N \geq 3$. This is in striking contrast to the corresponding result of Stein [2] stating that no peculiar polynomials with real coefficients all different from 0 , can exist for $N>4$. We have finally given upper bounds for the number of elements of these various constituent sets, which are presumably quite close to the actual values. Under an additional conjecture, we show that the cases of strict inequality numbered in (3.4) are actually the only ones, so that for all $N \geq 5$ the inequalities (3.3d, 3.3e, 3.3f) hold as equalities.

\section{Acknowledgment}

These findings were obtaining during the Gathering of Scientists held in November-December 2016 at the Centro Internacional de Ciencias (CIC) in Cuernavaca, Mexico. It is a pleasure to thank the participants for interesting discussions, and in particular Decio Levi for making a copy of [1] available to us. FL is further indebted to CONACyT grant Ciencias Básicas 254515 and UNAMPAPIIT-DGAPA grant IN103017 for financial support.

\section{References}

[1] S.M. Ulam, A collection of mathematical problems, Interscience, New York, 1960 (see pages 30-31).

[2] Paul R. Stein, "On polynomial equations with coefficients equal to their roots", Am. Math. Monthly 73 (3), 272-274 (1966).

[3] O. Bihun and F. Calogero, "Generations of monic polynomials such that the coefficients of each polynomial of the next generation coincide with the zeros of a polynomial of the current generation, and new solvable many-body problems", Lett. Math. Phys. 106 (7), 1011-1031 (2016); DOI: 10.1007/s11005016-0836-8. arXiv: 1510.05017 [math-ph].

[4] A. J. Di Scala and O. Maciá, "Finiteness of Ulam polynomials", La matematica e le sue applicazioni, Quaderni del Dipartimento di Matematica, Politecnico Torino, 11, July 2008, Turin, Italy; arXiv:00904.0133v1[math.AG]1April2009.

[5] O. Bihun and D. Fulghesu, "Polynomials Whose Coefficients Coincide with Their Zeros" arXiv:1705.02047v1[math.CA]

[6] Wolfram Research, Inc., Mathematica, Version 11.0, Champaign, IL (2016).

[7] G. Salmon Lessons Introductory to the Higher Modern Algebra, Cambridge University Press (1885), in particular Lesson VIII.

[8] See any standard textbook on algebraic geometry, for instance, Fulton, W. and Weiss, R. Algebraic curves: an introduction to algebraic geometry, Benjamin (1969) 